\section{LA VIVIENDA ANTE DESASTRES: UN ANÁLISIS DE LAS RESPUESTAS INSTITUCIONALES AÑOS DESPUÉS DE LA EMERGENCIA}

\author{
Aline Silveira Viana ${ }^{1^{*}}$
}

\section{RESUMEN}

En los últimos años, Brasil experimentó desastres de gran proporción relacionados a eventos hidrometeorológicos y climatológicos. Entre ellos, se destacan, los que ocurrieron en los estados de Santa Catarina y Rio de Janeiro, en los años de 2008 y 2011. El artículo busca reflexionar sobre las respuestas institucionales a la población afectada en los desastres, en el ámbito de las políticas habitacionales. Para eso, se ha realizado un estudio exploratorio-descriptivo, con el uso de la investigación documental y de entrevistas semiestructuradas. Entre los resultados, se ha observado que la solución estandarizada de los conjuntos habitacionales verticales puede ir contra los anhelos y derechos de vivienda digna de los afectados, en especial la de las personas de edad avanzada en las zonas rurales. Tales soluciones son reflejos de la manera de comprender el desastre y de los juegos de poder instalados en el territorio. Se concluye que, mientras los individuos y grupos no sean protagonistas en la decisión acerca del derecho a la vivienda digna, las políticas habitacionales en los desastres seguirán perpetuando obstáculos y vulnerabilidades históricamente construidos.

\section{PALABRAS CLAVES}

Adulto mayor, Vivienda social, Desastre, Práctica institucional

\section{HOUSING ISSUES AFTER DISASTERS: AN ANALYSIS OF INSTITUTIONAL RESPONSES AFTER YEARS FROM THE EMERGENCY}

\section{ABSTRACT}

Over the past few years, Brazil has experienced major disasters related to hydrometeorological and climatological events, such as those that happened in the states of Santa Catarina and Rio de Janeiro in 2008 and 2011. This study aims to analyzes the institutional measures related to housing policies to people affected in disasters. An exploratory-descriptive study was undertaken by using documentary research and semi-structured interviews. Among the results, it was found that standard solution of vertical housing complexes may work against the wish list and rights of those affected, especially, the rural older ones. These solutions reflect the way disasters are understood and are derived from power disputes in the territory. In conclusion, individuals and groups are apart from being protagonists in the decision about the rights to adequate housing, and public-housing policies are still perpetuating the barriers and vulnerabilities historically built.

\section{KEYWORDS}

Elderly, Public housing, Disaster, Institutional practice

\author{
1. Centro Nacional de \\ Monitoramento e Alertas \\ de Desastres Naturais \\ (Cemaden), São Paulo, \\ Brasil. \\ *Autor de correspondencia: \\ alinevianagerontologa@gmail. \\ com
}

Identificador: http://revistareder.com/ handle-o719-8477-2021-091

\section{RECIBIDO}

22 de enero de 2021

\section{ACEPTADO}

27 de febrero de 2021

\section{PUBLICADO}

1 de julio de 2021

\section{Formato cita}

Recomendada (APA):

Viana, A.S. (2021). La

Vivienda ante Desastres: Un Análisis de las Respuestas Institucionales Años Después de la Emergencia. Revista de Estudios Latinoamericanos sobre Reducción del Riesgo de Desastres REDER, 5(2), 6374. http://revistareder.com/ handle-0719-8477-2021-091

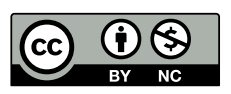

Todos los artículos publicados en REDER siguen una política de Acceso

Abierto y se respaldan en una Licencia CreativeCommons Atribución-NoComercial 4.0 Internacional.

Revista de Estudios Latinoamericanos sobre Reducción del Riesgo de Desastres (REDER)

Diseño: Lupe Bezzina 


\section{INTRODUCCIÓN}

Los desastres no son fenómenos recientes o esporádicos en Brasil. Entre 2003 y 2016, en aproximadamente $26 \%$ de los municipios brasileños, hubo cerca de 28.000 declaratorias federales de Situación de Emergencia (SE) y de Estado de Calamidad Pública (ECP), según el Sistema Integrado de Informaciones sobre Desastres (S2ID) de la Secretaría Nacional de Protección y Defensa Civil (SEDEC). El promedio anual en estos municipios fue de casi 2.000 declaratorias, un valor alarmante que, sin embargo, puede estar distante de la realidad. En 2013, en la última encuesta realizada, $49,53 \%$ de los municipios no disponen de coordinación de protección y defensa civil, como se dio a conocer en 2016 por la Confederación Nacional de Municipios (CNM) y $59,4 \%$ de los municipios no poseen un plan de gestión de riesgos (Instituto Brasileño de Geografía y Estadística [IBGE], 2013). Estos son factores que influyen en la capacidad del municipio de reconocer y actuar frente a los desastres.

En los últimos años, el país experimentó desastres de gran proporción relacionados a fenómenos hidrometeorológicos y climatológicos. Entre los más recientes, se destacan en esta investigación, los que ocurrieron en los estados de Santa Catarina (SC) y Rio de Janeiro (RJ), en los años de 2008 y 2011. Los municipios seleccionados en esta investigación fueron aquellos con el mayor número de fallecimientos, ocasionados por desastres en los años de 2008 y 2011. Coincidentemente, los municipios elegidos están entre los 213 municipios brasileños prioritarios del Plan Nacional de Gestión de Riesgos y Respuesta a Desastres para la gestión de riesgos y desastres por ser susceptibles de inundaciones y, entre los 161 con alta susceptibilidad de deslizamientos de tierra (Brasil, 2011).

La ocurrencia de desastres presenta una tendencia de crecimiento, factor que se corroboró con la ampliación del registro de SE e ECP por el sistema de protección y defensa civil (Universidad Federal de Santa Catarina [UFSC], 2013). De esta forma, se hace necesario ampliar y fortalecer las estrategias de prevención, mitigación, preparación, respuesta y recuperación frente a los desastres, en especial junto a los grupos considerados más susceptibles a fallecimientos en estos eventos, como el de personas con edad avanzada, cuyo crecimiento se da de manera expresiva. En 1940 había menos de un millón de ancianos en Brasil, solo seis décadas después llegamos a 9,3 millones. En 2025, tendremos la sexta población más grande de adultos mayores del mundo, con más de 32 millones de personas. La expectativa en 2050 es de 49 millones de ancianos, o sea, $19 \%$ de la población brasileña (IBGE, 2000; BRITO, 2007).

Es imprescindible incluir estos grupos en la planificación de acciones, porque cuando ocurren los desastres, la afectación se da de manera multifacética, comprendiendo varios aspectos de la vida biológica y social de estos individuos, lo que influye, entre otros aspectos, en las relaciones con la vivienda y el ambiente/lugar en que han vivido. En estudio anterior (Viana, 2020), por ejemplo, realizado con adultos mayores, familiares y miembros de la comunidad de un municipio afectado por un desastre relacionado a inundación y deslizamiento de tierra, la cuestión habitacional fue significativamente presente en las principales angustias enfrentadas, aun años después del fin de la vigencia de la declaratoria de emergencia.

Es imprescindible para la comprensión de la afectación, afirmar que el desastre se da en un tiempo social, resultado no solo del evento generador de la declaratoria federal, sino del solapamiento de varias crisis instaladas en el territorio, como la civilizacional, la crónica y la aguda (Valencio, 2012). Es así, aún mucho tiempo después de la declaratoria de SE o ECP, que el desastre puede seguir reproduciendo cuestiones en el territorio, en las condiciones de vida y de salud de la población afectada y en el tensionamiento de las relaciones de estos individuos con familiares, comunidad e instituciones.

Para mejor comprender las respuestas institucionales recibidas en el ámbito de las políticas habitacionales a los afectados, en especial al grupo de edad más avanzada, poco estudiado en el país. Bajo tales circunstancias, se realizó una investigación con gestores municipales directa o indirectamente involucrados en el registro, planeamiento y distribución de desarrollos de vivienda pública en cuatro municipios severamente afectados de Santa Catarina (SC) y Rio de Janeiro (RJ): Ilhota/SC, Blumenau/SC, Teresópolis/RJ y Nova Friburgo/RJ. 


\section{METODOLOGÍA}

Se trata de un estudio exploratorio-descriptivo, de carácter cualitativo, con el uso de la investigación documental y de entrevistas semiestructuradas, apreciada y previamente aprobada por el Comité de Ética en Investigación de la Escuela Nacional de Salud Pública Sérgio Arouca (ENSP) de la Fundación Oswaldo Cruz (FIOCRUZ), bajo el parecer nº 2.474.283.

El presente artículo es resultado de los hallazgos científicos de la tesis de doctorado de la autora, titulada 'Ancianos en los desastres - un análisis de las condiciones de vida, de los problemas de salud y de las respuestas sociales del poder público' (Viana, 2019). Se les preguntó sobre las medidas que se tomaron para el enfrentamiento de situaciones de desastres, las principales dificultades, los planes de enfrentamiento que se dispuso para auxiliar el segmento anciano de la población, entre otros temas, distinguiéndose entre los sectores de salud, defensa civil, asistencia social y consejo municipal del anciano. Aquí se presentan los principales hallazgos de la categoría vivienda.

Se realizaron entrevistas entre los meses de mayo y junio de 2018. Se entrevistó a secretarios(as) municipales - o técnicos(as) indicados(as) por aquellos - a las secretarias municipales de asistencia social, de salud, de defensa civil o responsables por el consejo municipal del anciano. Las citas se hicieron por email y teléfono. Todas las entrevistas se condujeron presencialmente.

Las entrevistas fueron conducidas después de la autorización de las secretarías municipales y del Consejo Municipal del Anciano, junto a la firma del Término de Consentimiento Libre y Esclarecido por los entrevistados. En total, fueron 18 entrevistados de cuatro municipios de Santa Catarina (SC) y Rio de Janeiro (RJ) - ver Tabla 1.

\begin{tabular}{|l|l|l|l|l|}
\hline & Ilhota/SC & Blumenau/SC & Teresópolis/RJ & Nova Friburgo/RJ \\
\hline Secretaria de Salud & 1 & 1 & 1 & 1 \\
\hline Secretaria de asistencia social & 1 & 1 & 1 & 1 \\
\hline Secretaria de defensa civil & 1 & 3 & 3 & 2 \\
\hline Consejo Municipal del Anciano & 1 & 1 & 0 & 1 \\
\hline
\end{tabular}

Tabla 1. Número de entrevistados por municipio Fuente: Autora, 2021.

La investigación documental incluyó las principales políticas de cada sector, tales como las Políticas Nacionales de: Protección y Defensa Civil, Asistencia Social, Salud del Anciano, el Estatuto del Anciano; los planes municipales de contingencia/enfrentamiento disponibles online e informaciones sobre la actuación de las secretarias estudiadas en los sitios institucionales y en periódicos electrónicos. Sin embargo, los documentos más interesantes para el diálogo con los análisis de las entrevistas fueron los extractos mediáticos, los que se incluyen en este artículo.

Los materiales colectados han pasado por el análisis documental y de contenido, bajo la interfaz de la mirada de la sociología de los desastres y de la gerontología. El análisis de las entrevistas se dio a través del análisis de contenido, a partir de la obra de Bardin 'Análisis de Contenido' del año de 1979, bajo el enfoque y los procedimientos descritos por Franco (2012). A partir de las cuestiones principales, se buscó emplear los procedimientos a seguir para describir el contenido de los mensajes de los entrevistados y posibles indicadores que posibiliten inferir o aproximar de las condiciones de producción social de las mismas.

Se inició con un preanálisis para identificar los documentos, los objetivos y los indicadores que juntos sostienen la interpretación de los mensajes (Franco, 2012). Se consideró las unidades de registro y de contexto en los análisis. Se indica que sean interrelacionadas para ampliar las posibilidades interpretativas incluso de los sentidos implícitos de las hablas, puesto que las unidades de contexto les dan significado a las unidades de análisis (Franco, 2012). En esta investigación, después de diversas lecturas de las entrevistas transcritas, se utilizó categorías a posteriori y unidades de registro del tipo tema, de acuerdo con la relevancia de las informaciones para comprender las respuestas sociales para el público anciano en los desastres, respetándose los criterios de agotamiento, homogeneidad y representatividad. Los resultados obtenidos en esta fase se complementaron por el contexto social, histórico, institucional y legal obtenido en la etapa de análisis documental, de modo a darle sentido y relación entre el habla y el espacio-tiempo estudiado. 


\section{RESULTADOS Y DISCUSIONES}

Los municipios estudiados disponen de una serie de registros de desastres desde sus fundaciones. Teresópolis/RJ y Nova Friburgo/RJ son municipios pertenecientes a la región serrana de Rio de Janeiro, de características geológicas y climáticas semejantes, de terrenos accidentados, pendiente fuerte y red de drenaje en fondos de valles. Semejante al observado en Blumenau/SC y llhota/SC, municipios pertenecientes de la mesorregión del Valle de Itajaí de Santa Catarina con presencia de sierras, topografía de fuerte pendiente y red de drenaje, formada principalmente por la cuenca del Río Itajaí (UFSC, 2013).

Según los datos del proyecto Base Territorial Estadística de Áreas de Riesgo (Tabla 2) en asociación con el Centro Nacional de Monitoramiento y Alertas de Desastres Naturales (CEMADEN), con fecha de abril de 2017, en Teresópolis/RJ, 28,0\% de la población residen en áreas de riesgo de inundaciones y desplazamiento de tierra. En Nova Friburgo/RJ, $18,5 \%$ de la población; en Blumenau/SC, 25,36\%; y en Ilhota/SC 49,25\% (IBGE, 2018). Específicamente sobre el percentil de ancianos, en el estudio conducido por Dias et al (2018) (Tabla 2), es estimada la población anciana en áreas de riesgo de deslizamientos e inundaciones. Para los municipios de Blumenau/SC y llhota/SC, sin embargo, no fueron encontrados estudios con esas informaciones.

\begin{tabular}{|l|l|l|l|l|}
\hline & Ilhota/SC & Blumenau/SC & Teresópolis/RJ & Nova Friburgo/RJ \\
\hline Población total & $12.355^{\star}$ & 309.011 & 163.746 & 182.082 \\
\hline Total de adultos mayores & $9,6 \%$ & $9,7 \%$ & $13,2 \%$ & $14,3 \%$ \\
\hline Población general en áreas de riesgo & $6.085^{\star *}$ & $78.371^{* *}$ & $45.772^{* *}$ & $33.660^{\star *}$ \\
\hline Adultos mayores en áreas de riesgo & - & - & $12,99 \%^{* * *}$ & $12,33 \%^{* *}$ \\
\hline
\end{tabular}

Tabla 2. Población total y población residente en áreas de riesgo de inundaciones y desplazamiento de tierra Fuente: Autora, 2021, basada en * IBGE (2010), **IBGE (2018), ${ }^{* * *}$ Dias et al. (2018).

Es importante decir que después del evento ocurrido en noviembre/2008 en Santa Catarina y en enero/2011 en Rio de Janeiro, los municipios están más organizados en lo que se refiere a la movilización de recursos. La movilización fue incluso, mencionada en algunas entrevistas de las secretarías de defensa civil. Según tres de los entrevistados, todo el proceso se agilizó gracias a una tarjeta que recibe repase inmediato y comprobación posterior, lo que apresuró la compra de artículos de emergencia. Como se sistematizó para Blumenau/SC en la investigación de Beckedorff (2017), por ejemplo, el municipio recibió recursos destinados a tres frentes principales.

En el primer frente, basado en la investigación de Schiochet (2009), a los estados afectados se puso a disposición por el ente federal, recursos de emergencia, por medio de la Medida Provisional $448 / 2008$ y de los decretos $n^{\circ} 6.663 / 2008$ y $n^{\circ} 6.688 / 2008$. Este último, incluso, facilitó el saque del Fondo de Garantía por Tiempo de Servicio (FGTS) a los residentes de los municipios afectados. En el segundo, se captó recursos para el suministro de asistencia financiera de BRL 415,00 (USD 72), el llamado 'Auxilio-Reacción', suministrado durante seis meses a las personas desplazadas, cuyo ingreso familiar fuera de hasta tres salarios mínimos (BRL 3300; USD 576) y que no estuvieran en refugios temporales. En el tercero, hubo recursos relacionados al suministro de albergues temporales y de reasentamiento para los conjuntos habitacionales (Beckedorff, 2017).

Todavía, en algunos municipios afectados por los desastres de noviembre/2008 (SC) y enero/2011 (RJ), según lo divulgado por la prensa y la Contraloría-General de la Unión hubo desvíos de recursos federales y de donaciones. En Teresópolis/RJ, por ejemplo, durante una investigación la Contraloría-General de la Unión (CGU), presentó irregularidades en la comprobación de cerca de BRL 7 millones (USD 1,2 milliones), lo que resultó en la casación del mandato del alcalde involucrado, como se informa en el sitio web de CGU "CGU y Defensa Civil Nacional identifican irregularidades en la aplicación de fondos transferidos a Teresópolis", el 08/05/2011 (Viana, 2020).

En varios municipios del estado de Santa Catarina hubo problemas con la entrega o la finalización de la construcción de viviendas por el Instituto Ressoar (un instituto con proyectos sociales de una red brasileña de televisión abierta - Record TV), conforme el reportaje publicado en 14/01/2019 por The Intercept Brasil. Según ese reportaje, la Record TV, por medio del Instituto Ressoar, habría recaudado fondos para las víctimas de inundaciones en Santa Catarina (BRL10,45 millones; USD 1,8 millones), prometido 700 casas, construido la mitad de ellas y cerrado sus actividades. En Blumenau/SC las 10 casas prometidas fueron finalizadas, en Ilhota/SC, 17 casas 
habían quedado inacabadas. Así, los afectados tuvieran que invertir recursos propios para la finalización o demolición, en resultado del deterioro de construcciones inconclusas.

Las discusiones sobre la aplicación de recursos y distribución de donativos inadecuadas también crean una situación, en el que los menos social y económicamente privilegiados entre los afectados en el desastre fueron los más atingidos (Valencio, Siena \& Marchezini, 2011). Valencio, Siena \& Marchezini (2011) presentan diversas situaciones experimentadas por los afectados de Santa Catarina durante la emergencia y dos años y medio después de ella. Lo que se constató fue "una mezcla de servicios públicos no prestados y otros de calidad cuestionable, además de la presencia de interacciones sociopolíticas degradantes (Valencio, Siena \& Marchezini, 2011, p.52)".

Según un reportaje publicado en 21/11/2015 en el diario online del G1 de Santa Catarina de la red Globo, en Ihota/SC, el ex-coordinador de la Defensa Civil había sido denunciado por el Ministerio Público de Santa Catarina por improbidad administrativa, puesto que había la sospecha de intercambio de viviendas por votos en las elecciones. Ante la situación, de las 100 familias registradas por la Compañía de Habitación del Estado de Santa Catarina, 66 no habían, hasta el año de 2015 , recibido las viviendas. Había denuncias en el reportaje sobre la entrega de viviendas para individuos no afectados en el municipio y sobre el uso de la vivienda para otras finalidades. Sin embargo, esas informaciones no fueron confirmadas en la presente investigación junto la Defensa Civil o la Secretaria de Asistencia Social del referido municipio. La conclusión de la denuncia no se ha encontrado en el Diario de la Unión o en sitios web especializados.

En Blumenau/SC, Samagaia y Angioni (2009) también identificaron situaciones inadecuadas y degradantes, en las que los siguientes derechos fueron violados, principalmente para con los desamparados en el municipio: el derecho de los usuarios de los servicios de asistencia social por el liderazgo, a manifestar sus intereses; el derecho al atendimiento digno, atento y respetuoso de los órganos públicos; el derecho de protección social de asistencia social; el derecho de equidad social y de manifestación pública; el derecho a igualdad en el acceso a la red de servicios sociales y asistenciales; el derecho al control social y defensa de los derechos socio-asistenciales.

Teresópolis/RJ presentó varios avances en el año 2019, si contrastados con el estudio anterior, realizado con ancianos y sus familias cinco años después de la crisis aguda (Viana, 2020). En la época, aún se discutía la construcción del viaducto para acceder al conjunto habitacional, la entrega de viviendas a los afectados y la necesidad de asistencia a estos ciudadanos. En las reivindicaciones presentadas en las manifestaciones públicas, como las que ocurrieron en enero de 2013 y 2015 en Teresópolis/RJ (acompañadas en locus), el poder público había fracasado en la cuestión habitacional, en la recuperación de puentes y carreteras, en la conclusión de las inspecciones, en el pago de las indemnizaciones, así como en el suministro de soporte biopsicosocial a las familias de los desaparecidos y a los afectados en general (Viana, 2020).

En esta última incursión en campo en mayo de 2018, por otro lado, la entrega de las viviendas había ocurrido para la mayor parte de los afectados, según informó la Secretaría de Desarrollo Social. Lo mismo se observó para los demás municipios, donde la mayoría de los afectados ya había sido dirigida para los conjuntos habitacionales o a las indemnizaciones. Ilhota/SC fue el único municipio en confirmar que, la Secretaría Municipal de Asistencia Social, suplió la totalidad de la demanda por viviendas por parte de los afectados en noviembre de 2008 y actualmente, en 2018, dispondría de recursos para sanar el déficit habitacional del municipio. En Nova Friburgo/ $\mathrm{RJ}$, la solución de retirada fue rechazada por los residentes del barrio Córrego D'antas y debido a la presión y a la movilización, los ciudadanos han logrado obras que garantizaran su seguridad en permanecer en el local anteriormente afectado. La discusión sobre el derecho a vivienda necesita ser debatida junto a la comunidad, porque, como lo puso uno(a) de los(as) entrevistados(as):

"Las personas no son como tazas que puedes quitarlas de un sitio y ponerlas en otro... las consecuencias de esa política [de habitación] es inhumana. [...] Tuvimos personas que fueron afectadas que eran de la zona rural, pequeños productores rurales. Creo que es un estudio que hay que hacer, porque hoy esas personas que vivían de la tierra están dentro de un apartamento minúsculo y no tienen ningún contacto con la tierra. [...] Hay casos de personas que viven en estas unidades y tienen gallinas en el apartamento, porque es el hilo de vínculo que se mantiene de la vida que tenían antes. Su vida era la cría de cerdos, la cría de gallinas, plantar, estar en contacto con la tierra. La cuestión de la renta, lo de 
regalar la casa es paliativo, ¿qué casas, qué unidades habitacionales son estas? La gente está acostumbrada a vivir en una casa simple, de un cómodo tal vez, pero que ha sido de sus abuelos, tatarabuelos, han crecido y vivido ahí, con el pie en el suelo, y entonces pones estas personas en un apartamento de poquísimos metros cuadrados... Es el momento de aprovechar la situación, porque hoy no hay una solución, hay que pensar soluciones que garanticen la dignidad de estas personas. Nadie vive en un área de riesgo porque así lo quiere [...] el poder público necesita tener esa mirada y no culpar a los que son víctimas de la planificación que sucedió hace años." [Entrevistado(a) S18].

El proceso de decisión entre el regresar al área afectada o aceptar nuevas condiciones habitacionales, no necesariamente mejores o equivalentes al anterior, comprende varios elementos, como lo pondera Henry (2013). En el estudio norteamericano (2013) conducido con adultos mayores evacuados, profesionales y voluntarios, había elementos objetivos y subjetivos en el proceso de decisión. Entre ellos, estar o no de acuerdo con la proposición oficial/técnica, saber el área geográfica donde se ubica (o se ubicará) la vivienda, quien debe tomar la decisión y lo que es el mejor para el anciano o para la familia.

En la mayoría de los municipios, conforme se resaltó en las entrevistas, ancianos, incluso de municipios vecinos, migran después de la jubilación para áreas rurales de estas localidades en busca de mejor calidad de vida y tranquilidad, al mismo paso que miembros jóvenes y adultos de la familia se van de estas áreas en busca de trabajo en municipios más grandes o centros urbanos. El movimiento migratorio urbano-rural relacionado a la actividad productiva ya está bien descrito en la literatura (Garbaccio, Tonaco, Estêvão \& Barcelos, 2018).

Otra característica es la de ancianos nacidos y criados en estas áreas, en tierras ya pertenecientes a la familia por generaciones, de ingresos provenientes del trabajo en el campo. El mayor contingente de hombres mayores en el medio rural en los municipios sigue un comportamiento nacional, por permanecer a trabajar en el campo, mientras las mujeres mayores migran con los hijos a las áreas urbanas para ayudar, por ejemplo, en el cuidado de los nietos. (IBGE, 2018; Garbaccio, Tonaco, Estêvão \& Barcelos, 2018).

En la investigación junto con los municipios, según las entrevistas, el permanecer en el local afectado, principalmente en el área rural se dio justamente por tratarse de locales pasados entre las generaciones, con vínculos culturales, familiares, afectivos y de medio de trabajo. En los relatos, la permanencia de la población en el medio rural afectado también sucedió por no tener alternativas que permitieran calidad de vida equivalente o mejor de lo que ya tenían viviendo en estas localidades.

Las características de los ancianos rurales de estos municipios, sumado a la dificultad de cobertura efectiva de los servicios en el territorio, influyen en las formas en que los ancianos, comunidades y servicios pueden prepararse para situaciones de emergencia y calamidad pública. En la literatura, vemos que el nivel de ingreso, de escolaridad, de dependencia y de funcionalidad, así como tener acceso a fuentes de información de alerta y vivir con otros familiares, son significativos para la condición de preparo de los ancianos y familiares en el enfrentamiento de los desastres (Al-Rousan, Rubenstein \& Wallace, 2014; Luo, Cong \& Liang, 2015). Tales elementos también pueden estar presentes en el contexto de los municipios estudiados, siendo necesario nuevas investigaciones para la detección de los factores que influyen la capacidad de preparación y evacuación en áreas más aisladas, como las rurales aquí discutidas, cuando hay mayor dificultad de respuesta rápida de los servicios públicos.

Por otro lado, la solución estandarizada de los conjuntos habitacionales verticales (Figura 1) en áreas periféricas pueden ir contra los anhelos y derechos de vivienda digna a los afectados por producir condiciones de perpetuación y agravamiento de la vulnerabilidad social como han indicado todas las secretarías involucradas en la gestión de estos conjuntos. 0 incluso los conjuntos horizontales, sin infraestructura de recolección de aguas residuales, como alerta Albino (2016), basado en las casas entregadas del Programa COHAB (Empresa de vivienda), en Braço do Baú (llhota/SC).

"Evaluando todo el proceso, el Minha Casa, Minha Vida fue construido con un número muy grande de apartamentos, hay muchas personas viviendo ahí...[...] hay muchos problemas,
Minha casa Minha Vida, creado en 2009 (MCMV) es el mayor programa habitacional en Brasil. El programa de Gobierno Federal tiene como objetivo principal facilitar el acceso a vivienda para las familias brasileñas de bajos ingresos. 
de tráfico, problemas sociales [...] las familias más pobres, más vulnerables también fueron atingidas y ellos se juntaron, de varios barrios, tanto en las viviendas provisionales como en las residenciales. Las más numerosas, las prioridades llegan primero. Los primeros en ir al residencial, es hoy el que más problemas tiene. Hay un poco más de 300 apartamentos. Hoy ya es diferente. Con el Minha casa Minha Vida que es planteado hoy, tiene un máximo de 50 apartamentos. El conjunto ya está planeado en bloques pequeños, volcados de manera que las ventanas no se... ¿me entiendes? Es otra lógica. En el otro no, los apartamentos están todos muy cerca uno del otro, este volcado al aquel [...] Si contrastamos el primero residencial con el último es notable la diferencia. [...] porque los riesgos allá quedan mucho más evidentes. [...] los residenciales nuevos ya están planeados con terreno próximo para la infraestructura, diferente de los anteriores." [Entrevistado(a) S11].
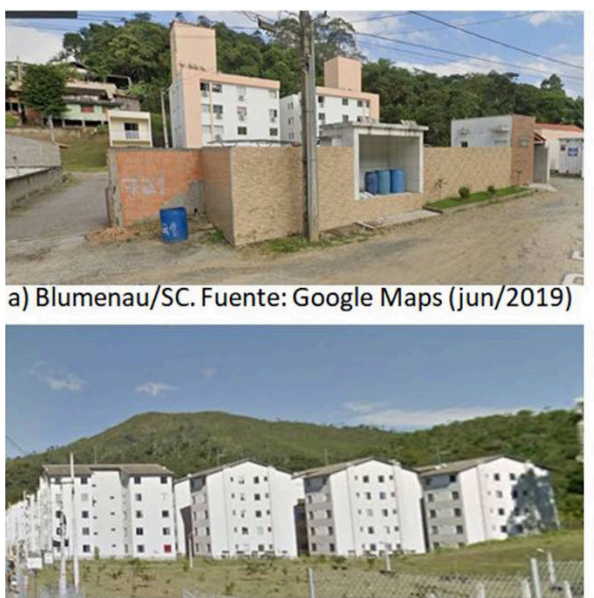

b) Teresópolis/RJ. Fuente: Google Maps (may/2019)

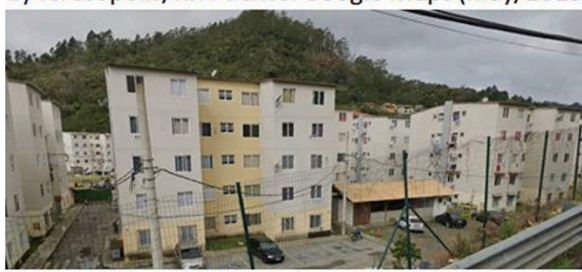

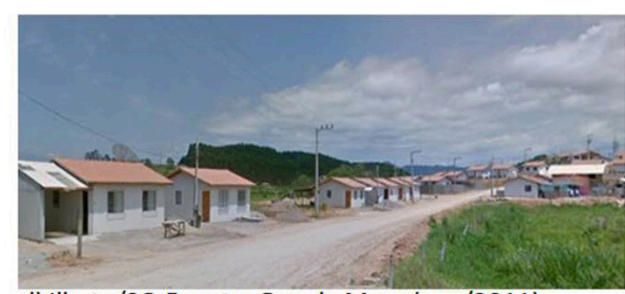

d) Ilhota/SC. Fuente: Google Maps (nov/2011)

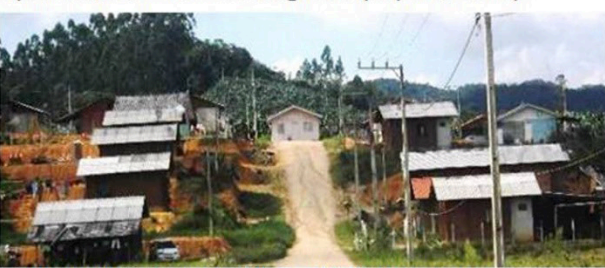

e) Ilhota/SC. Fuente: Albino (2016), registrada por Lisangela Albino em 2013.

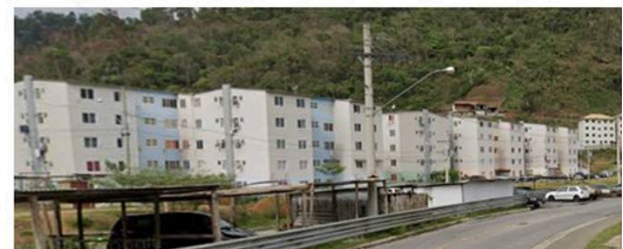

c) Nova Friburgo/RJ. Fuente: Google Maps (oct/2019) f) Nova Friburgo/RJ. Fuente: Google Maps (oct/2019)

Figura 1. Viviendas entregadas a los afectados en Blumenau/SC, Teresópolis/RJ, Nova Friburgo/RJ y Ilhota/SC Fuente: Google Maps (2011, 2019); Albino (2016)

Como ha indicado el estudio realizado por Portella y Oliveira (2017, p. 512) el Programa Minha Casa Minha Vida es el principal modelo habitacional en el país para los desamparados en los desastres que "el gobierno federal insiste en llamar de política habitacional". El proyecto y la ejecución, como explanan los autores, es realizado por grandes constructoras y contratistas, cuyo fin es la estandarización y la reducción de gastos en detrimento del respeto al modo de vida y cultura de estas personas, sus vínculos con el lugar, la tierra y la comunidad.

Es imprescindible decir que la manera como se comprende el desastre influye en las estrategias a ser adoptadas que pueden ser inclusivas o no alineadas con las necesidades y problemas enfrentados por los directamente afectados, tales como los relacionados a la vivienda, aquí discutidos. Como indica la literatura, la concepción de desastre varía no sólo entre las diferentes áreas del conocimiento, sino también dentro de las mismas áreas, instituciones e instancias de poder (Quarantelli, 1998; Oliver-Smith, 1998). La falta de intersección analítica entre las lineas teóricas y las áreas que estudian los desastres, por ejemplo, así como el aumento en la complejidad en la comprensión de los desastres, dificultan el establecimiento de un consenso sobre el concepto de desastres (Quarantelli, 1998). Sin embargo, en el medio institucional brasileño, es recurrente la comprensión y actuación vertical, centrada en el factor técnico y en el medio físico (las amenazas) (Valencio, 2012).

Los desastres se expresan como un proceso social y como tal, es marcado por el tiempo social, que no termina simplemente con la pérdida de la vigencia de la declaratoria que lo reconoció. Así, la naturalización de los desastres y el tratamiento únicamente tecnicista y cronológico deslegitima 
el debate político y social de la responsabilización de los diferentes actores sociales entorno la producción, mantenimiento y enfrentamiento de los desastres (Quarantelli, 1998; Valencio, 2012). Se hace necesario oponerse al discurso que establece el elemento físico relacionado al desastre (lluvia, terremoto, tempestad etc.) como el principal responsable por el desastre en sí mismo, así como el discurso que las respuestas institucionales necesitan ser guiadas solamente en el elemento físico, no actuando sobre el contexto social, cultural, histórico, ambiental y económico que constituye la producción social del desastre.

La participación de los diferentes grupos involucrados es fundamental para romper con soluciones que no dialogan con la realidad de los afectados. Obstáculos para la participación social en las políticas públicas y acciones de prevención reflejan de diferentes formas en las comunidades. A causa de los desastres, los ciudadanos pasan a ser afectados con carencias negociables, objeto de procesos burocráticos lentos y aplanadores de la condición humana en estos contextos (Valencio; Siena \& Marchezini, 2011).

En los municipios estudiados, muchas iniciativas de auxilio se han dado después que las comunidades afectadas se unieron, lo que expresa los procesos de consciencia y conducta a nivel colectivo, por medio de la participación y organización (Castellanos, 1991). Aquí se destacan tres iniciativas de los municipios estudiados, la Asociación de las Víctimas de las Lluvias de 21 de enero de 2011 en Teresópolis (AVIT) recientemente disuelto, la Associação de Moradores do Bairro Córrego D'Antas en Nova Friburgo/RJ todavía en actividad y el Movimiento de los Atingidos por el Desastre (MAD) en Blumenau/SC sobre el que no se ha identificado acciones recientes.

Los grupos mencionados se convirtieron en medios importantes de resistencia contra las arbitrariedades públicas cometidas y de protagonismo en las soluciones propuestas, aunque haya muchos obstáculos, internos y externos al grupo. Como ejemplo de este último, hay la morosidad de los procesos burocráticos.

"Da igual, todo el municipio está en área de riesgo. Hay que unirse y discutir de manera más profunda. La gente de Córrego D'antas así lo hizo... "Habrá que removerlos todos" y luego se han organizado. Hubo una situación en que el gobernador ahí estaba y la asociación ha presionado y luego hicieron una obra gigantesca de contención de laderas. Sinceramente, creo que este es el camino, discutir el mantenimiento de estas personas. Discutir de manera profunda la política de habitación social, de dignidad de vivienda, de seguridad humana. Nos sacan de un área de riesgos para ponernos en otra [social]" [Entrevistado(a) S18].

En Nova Friburgo/RJ la asociación ha ganado el apoyo de universidades, de centros de investigaciones y de la propia comunidad. Han alcanzado varios resultados positivos, entre ellas las obras de contención, el Centro Sociocultural, la implantación de la Red para la Gestión de Riesgo de la Cuenca del Arroyo D'Antas (Reger-CD), la retirada de una incineradora de residuos infectantes y un sistema de comunicación vía radio amador, como fue difundido por el Diario a Voz da Serra (22/08/2018) y contenido en los relatos de investigadores miembros de la Reger-CD en seminarios del área, en la que se hubo la oportunidad de participar.

En Teresópolis/RJ, la Asociación de Víctimas (AVIT) se movilizó intensamente para la garantía de renta social, indemnizaciones y/o vivienda a los desamparados. Se realizó protestas y manifestaciones, asesorías jurídicas, audiencias, participaciones en eventos científicos con publicación, incrementó el acceso de atención médica, odontológica, psicológica y la proposición de alteraciones en la ley orgánica del municipio para la aplicación de ingresos en el Fondo Municipal de Habitación de Interés Social (Diario Net Diário, 19/02/2018; Carneiro, 2015).

En Blumenau/SC, en el primer año, se han identificado actividades del Movimiento de los Atingidos por el Desastre (MAD), que se enfocaron en el acceso a vivienda. La última actividad identificada fue en 2009 y su permanencia fue mencionada en julio de 2015 en el Diario de Justicia del Estado de Santa Catarina, en un proceso judicial de reintegración/mantenimiento de propiedad en contra los miembros que habian ocupado propiedades después de noviembre de 2008. La decisión, sin embargo, había sido favorable a los ocupantes hasta que las soluciones habitacionales fueran tomadas. De esta manera, el Movimiento ha logrado acceso a la principal reivindicación. 
Sin embargo, la manera como las soluciones habitacionales fueron presentadas a los municipios, acabaron por generar nuevos problemas a los afectados y a los servicios públicos. En un reportaje realizado en 04 de marzo de 2018 en Blumenau/SC, en consonancia a los relatos obtenidos durante las entrevistas, la reportera de noticias Marina Estarque en entrevista con ancianos y familias residentes en los conjuntos habitacionales, encontró relatos sobre las varias incursiones policiales debido a la acción del tráfico en estos locales, así como el miedo y la inseguridad constante de los residentes. Esto se refleja en los apartamentos con rejas en las ventanas, en el abandono de apartamentos, en el estigma sufrido incluso en la búsqueda de empleo por ser residente de estos conjuntos y en el deseo de vender los apartamentos y buscar otras localidades (Folha De S. Paulo, 14/03/2018).

En el referido municipio, Peixer (2014) se ocupó sobre cómo los espacios de uso privado de los conjuntos habitacionales financiados por el Programa Minha Casa Minha Vida, dirigido a los afectados en el desastre impactaban el cotidiano de las familias que residían en estas viviendas. Se evaluó diversos aspectos y, de manera general, el principal resultado fue que ese modelo habitacional adoptado se direcciona a los mínimos establecidos por el programa nacional, pero de manera dislocada con el contexto de uso, cultura y modo de vida de las familias, perjudicando el regreso a la vida cotidiana anterior al desastre. Uno de los puntos discutidos es la distancia de los conjuntos de los centros comerciales e industriales de trabajo de esas personas.

Conforme señalan los entrevistados, se hace necesario repensar la solución habitacional, así como las intervenciones del ente público en los conjuntos ya entregados, porque hay el aumento de demanda de servicios de asistencia social y de salud de modo no compatible a los disponibles en la red local.

"Se creó un condominio [...] donde se puso familias referenciadas en todos los barrios. Es un espacio en donde hay una determinada cultura y se puso familias de otras culturas, otros valores. Hay familias de la zona rural, familias no tan rurales, pero con valores totalmente distintos de aquel territorio. Hubo un aumento en los casos de violencia, casos de niños acompañados por el Consejo Tutelar. Hay, lo que antes no había, 10.000 familias referenciadas bajo la atención de un mismo $\mathrm{CRAS}^{2}$, que son los 10.000 individuos en el condominio. Esas personas existían, cada una en su región de origen, sin una demanda específica para la asistencia. Hoy el condominio es 100\% demanda para la asistencia social" [Entrevistado(a) S14].

En Nova Friburgo/RJ, el conjunto habitacional Terra Nova fue entregado a los afectados años antes que en Teresópolis/RJ. Sin embargo, los ciudadanos cuestionan las condiciones de vivienda en este conjunto habitacional, como presentado en el Seminario Internacional de Desnaturalización de los Desastres y Movilización Comunitaria, realizado en octubre de 2015 en el Rio de Janeiro/ RJ (acompañado en locus) y posteriormente también relatado en el estudio de Portella y Oliveira (2017). En el caso de familias numerosas, intergeneracionales y con agregaciones, cerca de $44 \mathrm{~m}^{2}$ de espacio construido puede no condecir con el mínimo comprendido por ellas para el desarrollo de su habitus y de su vida cotidiana. $Y$ el hecho de vivir en apartamentos puede no condecir con los anhelos y con la historia de algunas familias, como presentado por los afectados en el referido Seminario.

Discutir la categoría vivienda en estos contextos de desastres nos lleva a reflexionar el significado de la pérdida del hogar, de los desplazamientos, de los cambios de relaciones comunitarias en el ámbito del envejecimiento. Como nos recuerda Bachelard (1988), la vivienda, así como cada objeto y lugar más pequeño dentro de esta tiene significados para el residente, representando refugio, acogimiento, protección y tranquilidad. La pérdida de la vivienda, de los objetos y de la seguridad física y emocional que proporcionan, en el caso de la persona de edad avanzada puede significar una ruptura brusca con los elementos que a conectaban con su historia relacionada con la de los objetos y de las personas que la compusieron, porque como nos recuerda Tuan (1983, p.154), estos están presos al lugar [...], a las personas, a los recursos de la comunidad y uno al otro.

En el caso de los municipios visitados, los conflictos generados con la agregación de personas provenientes de diferentes localidades de la ciudad, de diferentes perfiles y conductas (sin un gerenciamiento adecuado de los conflictos), hacen con que la solución habitacional adoptada
2. El CRAS es una entidad pública estatal descentralizada de la política de asistencia social, siendo responsable por la organización y oferta de los servicios socio asistenciales de la Protección Social Básica del Sistema Unico de Asistencia Social (SUAS) en las áreas de vulnerabilidad y riesgo social de los municipios y distrito federal. 
tenga se convertido en un problema a los afectados y a la gestión municipal. En Nova Friburgo/RJ, por ejemplo, los residentes de estos conjuntos enfrentan una vez más problemas con inundaciones en la localidad, perjudicando principalmente a los residentes del primer piso, en la mayoría de los casos ancianos o deficientes físicos. Se suma a eso, el no planteamiento efectivo y entrenamiento institucional de evacuación a los grupos más vulnerables, como los ancianos.

Lo que es un serio problema, ya que estos pueden no lograr evacuar el local o irse para los pisos superiores en caso de emergencia. Conforme lo visto en la literatura, tal escenario puede llevar a graves consecuencias para la sobrevivencia y la mantenencia de la salud de ancianos cuando hay obstáculos para la rápida evacuación (Jangi, 2012; Langan \& Palmer, 2012; Bei et al., 2013).

Las acciones públicas en estos emprendimientos todavía son insuficientes en la mayoría de los municipios estudiados, como lo evidencia uno(a) de los(as) entrevistados(as).

"Todo tiene dos polos, el malo es que el [conjunto habitacional], se convirtió en una fuente de 'votos fáciles' para el [nombre omitido], pero por otro lado hay una intervención mínima en el estado de las cosas, de una manera no muy adecuada, pero al menos están ahí... así conseguimos obtener algunas acciones. Es un lugar gigantesco. En la entrada estaba empezando a haber comercio ilegal, lo que es muy favorable a un posible tráfico... La autoridad local estuvo ahí, hubo fiscalización, había de todo... Pero asi es, las personas que no tienen acceso a trabajo se arreglan como pueden. El [órgano estadual] estar ahí, el CRAS estar ahí es un intento muy tímido, pero aun así es algo. Las personas comprenden que el estado ahí está de alguna manera." [Entrevistado(a) S18].

Hay varios obstáculos, no solamente en estas localidades, como lo enlistó Carneiro (2015) en la investigación conducida en Teresópolis/RJ acerca del uso y valores insuficientes del auxilio para la renta. Como lo relató la investigadora Valencio (2014) basada en años de investigación en el campo en el país, sobre las limitaciones del auxilio para la renta, se concluye que necesita ser reevaluado. La autora expone la discriminación del mercado inmobiliario contra las familias numerosas y con niños, las pocas opciones de viviendas fuera del área de riesgo compatible con el valor de la asistencia financiera recibida, la inflación de los precios del mercado inmobiliario local, lo que impacta en los recursos para otros aspectos básicos de la vida de los afectados, y por fin, la duración de los alquileres generalmente restricta e inferior al tiempo de espera por otras soluciones habitacionales.

Como ha enlistado Marchezini (2014), basado en el desastre ocurrido en el interior de São Paulo, hay prácticas, mecanismos y discursos en el ámbito biopolítico que influyen la vida cotidiana y los procesos de reconstrucción y recuperación de los afectados en los desastres. Los resultados encontrados parecen venir en contra, al menos en parte, de algunas prácticas/ ausencias identificadas en las entrevistas y documentos aquí levantados, como la preocupación del ente público de "hacer vivir" en la crisis aguda, y de "dejar morir" a lo largo de los años con el olvido/no-priorización de estos, lo que demanda un esfuerzo continuado en un "hacer resistir" individual y colectivo/comunitario de los afectados, delante de las arbitrariedades del ente público, en los diferentes niveles de poder.

Como se ha observado, fueron y siguen siendo realizados esfuerzos en el ámbito municipal para el enfrentamiento del desastre, no necesariamente respaldado por los entes federales y estaduales a largo plazo, salvo acciones puntuales técnicas y verticales conforme lo visto en la mayoría de los municipios.

\section{CONSIDERACIONES FINALES}

Los desastres acaban por ser marcos importantes para que se repiense los padrones constructivos y las políticas habitacionales existentes. Soluciones habitacionales humanizadas y participativas en caso de desamparo necesitan ser priorizadas en las acciones de gestión de riesgo, así como las de planeamiento conjunto entre las áreas de asistencia social, de obras y defensa civil. La falta de diálogo con los que se beneficiarán de estas soluciones preestablecidas, acaba por provocar situaciones como las descritas a lo largo de esta investigación.

Estrategias de mitigación y reducción de riesgo que permitan la permanencia de los residentes locales en sus comunidades, si así lo desean, puede abarcar las políticas habitacionales en caso 
de desastres. Obras para mejorar la estabilidad del terreno, contener movimiento de piedras y masa, mejorar el drenaje del suelo y la protección de los cursos de agua, entre otros, pueden ser soluciones viables para la no reubicación de los afectados y la destinación para conjuntos habitacionales estandarizados que están distantes de la realidad y habitus de estos individuos. El repensar de manera colectiva aún está lejos de concretizarse como se observó en los municipios estudiados, aún más, cuando se trata de grupos de personas de edad avanzada que fueron desplazadas. Aquí, cabe destacar que el derecho a la vivienda digna es constitucional, posible y, por tanto, no utópica. En lo que atañe a la persona de edad avanzada afectada, cabe a la unión, estados, municipios y sociedad garantizar que tal derecho sea respetado.

\section{AGRADECIMIENTOS}

El presente estudio fue realizado con el apoyo del Consejo Nacional del Desarrollo Científico y Tecnológico (CNPq). Deseo agradecer el apoyo del CNPq a lo largo del proceso de doctoramiento, del que deriva el presente artículo, así como la orientación del Prof. Dr. Carlos Machado de Freitas.

\section{REFERENCIAS}

Albino, L. (2016). O desastre em meio rural: análise dos impactos socioeconômicos na região dos baús, Ilhota, SC. 2014. Tesis (Mestrado em Planejamento Territorial e Desenvolvimento Socioambiental) Universidade do Estado de Santa Catarina, Florianópolis.

Al-Rousan, T.M., Rubenstein, L.M., \& Wallace, R.B. (2014). Preparedness for natural disasters among older US adults: a nationwide survey. American Journal of Public Health, 104(3), 506-511. https://doi. org/10.2105/AJPH.2013.301559

Bachelard, G. (1988). A poética do espaço. Tradução Antônio de PaduaDanesi; revisão da tradução Rosemary Costhek Abílio. São Paulo: Martins Fontes.

Beckedorff, F.F. (2017). Do deslocamento involuntário ao assentamento arbitrário: políticas habitacionais pós desastre em 2008, Blumenau, Santa Catarina. Tesis (Mestrado em Desenvolvimento Regional). Universidade Regional de Blumenau.

Bei, B., Bryant, C., Gilson, K.M., Koh, J., Gibson, P., Komiti, A., Jackson, H., \& Judd, F. (2013). A prospective study of the impact of floods on the mental and physical health of older adults. Aging $\mathcal{E}$ mental health, $17(8)$, 992-1002.

Brasil. (2011). Ministério das Minas e Energia (MME). Mapa Previsional da Suscetibilidade a Enxurradas dos Municípios Brasileiros. CPRM - Serviço Geológico Do Brasil. http://www.cprm.gov.br/publique/ media/mapa_Suscet_Desliz_Enxur_Brasil.pdf

Carneiro, C. (2015). Memória da Associação das vítimas das chuvas de Teresópolis/RJ. In: Siqueira, A. et al. (Orgs). Riscos de desastres relacionados à água. Aplicabilidade de bases conceituais das ciências humanas e sociais para a análise de casos concretos. São Carlos: RiMa Editora, p. 431-436.

Castellanos, P.L. (1991). Sistemas nacionales de vigilancia de la situación de salud según condiciones de vida y del impacto de las acciones de salud y bienestar. Organización Panamericana de La Salud.

Confederação Nacional de Municípios - CNM. (2016). Defesa Civil e Prevenção de Desastres: Como seu Município pode estar preparado-Coletânea Gestão Pública Municipal: Gestão 2017-202o. Brasília: CNM.

Dias, M.C.A., Saito, S.M., Alvalá, R.C.S., Stenner, C., Pinho, G. \& Nobre, C.A. et al. (2018). Estimation of exposed population to landslides and floods risk areas in Brazil, on an intra-urban scale. International Journal of Disaster Risk Reduction, 31, 449-459. https://doi.org/10.1016/j. ijdrr.2018.06.002

Franco, M.L. (2012). Análise de conteúdo. 4a ed. Brasília: Liber Livro.

Garbaccio, J.L., Tonaco, L.A.B., Estêvão, W.G. \& Barcelos, B.J. (2018). Aging and quality of life of elderly people in rural areas. Revista Brasileira de Enfermagem, 71(Suppl. 2), 724-732. https://doi. org/10.159o/0034-7167-2017-0149

Henry, J. (2013). Return or relocate? An inductive analysis of decision-making in a disaster. Disasters, 37(2), 293-316. https://doi.org/10.1111/j.1467-7717.2012.01303.x

Instituto Brasileiro de Geografia e Estatística- IBGE. (2018). População em áreas de risco no Brasil. 1 ed. Rio de Janeiro/RJ: IBGE. https://biblioteca.ibge.gov.br/visualizacao/livros/liv101589.pdf

Instituto Brasileiro de Geografia e Estatística - IBGE. (2013). Perfil dos Municípios Brasileiros. IBGE. 
Jangi, S. (2012). Facing uncertainty-dispatch from Beth Israel Medical Center, Manhattan. N Engl J Med, 367(24), 2267-2269. https://doi.org/10.1056/NEJMp1213844-

Langan, J.C. \& Palmer, J.L. (2012). Listening to and learning from older adult Hurricane Katrina survivors. Public Health Nurs, 29(2), 126-35. https://doi.org/10.1111/j.1525-1446.2011.00996.X_

Luo, J., Cong, Z., \& Liang, D. (2015). Number of warning information sources and decision-making during tornadoes. American journal of preventive medicine, 48(3), 334-337. https://doi.org/10.1016/j. amepre.2014.09.007

Marchezini, V. (2014). Janeiro de 2010, São Luiz do Paraitinga/SP: lógicas de poder, discursos e práticas em torno de um desastre. Tesis (Doutorado em Ciências Humanas) - Universidade Federal de São Carlos, São Carlos.

Narváez; L., Lavell, A. \& Ortega, G.P. (2009). La gestión del riesgo de desastres: un enfoque basado en procesos. San Isidro: Secretaría General de la Comunidad Andina.

Oliver-Smith, A. (1998). Global changes and the definition of disaster. In: Quarantelli, E.L. (Ed.), What is a disaster? Perspectives on the question. Routledge: London \& New York, p. 177- 194.

Peixer, K.T. (2014). Programa minha casa minha vida: adequação dos projetos às características das famílias moradoras. O caso de Blumenau/SC e a resposta ao desastre de 2008. Tesis (mestrado) Universidade Federal de Santa Catarina. Florianópolis/SC.

Portella, S. \& Oliveira, S.S. (2017). Vulnerabilidades deslocadas e acirradas pelas políticas de habitação: a experiência do Terra Nova. In: Marchezini, V., Wisner, B., Londe, L.R., \& Saito, S.M. (Eds.), Reduction of vulnerability to disasters: from knowledge to Action. São Carlos: RiMa Editora, p. 499-516.

Quarantelli, E.L. (Ed.). (1998) What is a disaster? Perspectives on the question. London \& New York: Routledge, p. 234-273.

Samagaia, J. \& Angioni, M. (2009). Situação dos desabrigados/atingidos pelo desastre e a assistência (des)organizada. In: Frank, B.; Sevegnani, L. (Eds.), Desastre de 2008 no Vale do Itajaí. Água, gente e política. Blumenau: Agência de Água do Vale do Itajaí, p. 139-147.

The Intercept Brasil. (2019, 14 de enero). Calote: Record captou R\$ 10mi para vítimas de enchentes em SC, prometeu 700 casas, construiu metade e sumiu. https://theintercept.com/2019/o1/13/ record-casas-enchente-sc/

Tuan, Y.F. (1983). Experiências íntimas com o lugar. In: Tuan, Y.F. (Ed.), Espaço e lugar:a perspectiva da experiência. Tradução Lívia de Oliveira. São Paulo: DIFEL, p. 151-219.

Universidade Federal De Santa Catarina - UFSC. (2013). Atlas brasileiro de desastres naturais 1991 a 2012: volume Rio de Janeiro e volume Santa Catarina. Florianópolis: Centro Universitário de Estudos e Pesquisas sobre Desastres, UFSC.

Valencio, N.F.L S., Siena, M. \& Marchezini, V. (2011). Abandonados nos desastres: uma análise sociológica de dimensões objetivas e simbólicas de afetação de grupos sociais desabrigados e desalojados. Brasília: Conselho Federal de Psicologia.

Valencio, N.F.L.S. (2014). Desastres no Brasil: a face hídrica do antidesenvolvimento. In: Valencio, N.F.L.S. \& Siena, M. (Eds.), Sociologia dos desastres - construção, interfaces e perspectivas (Vol. 3). São Carlos, RiMa Editora.

Valencio, N.F.L.S. (2012). Para Além do 'Dia do Desastre'. Curitiba: Editora Appris.

Viana, A.S. (2020). O Desastre e o Caos Velado: O enfrentamento individual, interpessoal e coletivo de idosos e familiares (2 Ed). Newcastle: RedWaterlat, EDUEPB, CICCUS, IELAT. 